\title{
Non-smooth Hybrid Energy Functional Regularization Model for Image Reconstruction
}

\author{
Xu-Chao LI ${ }^{1, a}$, Song-Yan MA ${ }^{1}$ and Wen-Juan $\mathrm{LI}^{2}$ \\ ${ }^{1}$ College of Computer and Information Engineering, Chifeng University, Chifeng, Inner Mongolia, 024000, China \\ ${ }^{2}$ College of Mathematics and Statistics, Chifeng University, Chifeng, Inner Mongolia, 024000, China
}

\begin{abstract}
For overcoming the shortcomings of total variation for image reconstruction, which easily smooth image texture, and produce lots of artificial edges, a non-smooth hybrid energy functional regularization model and iterative algorithm is proposed. Firstly, fitting term is described by $\mathrm{L}_{1}$ norm for blurred image by system and salt and pepper noise, regularization terms are described by fractional order bounded variation function semi-norm and $\mathrm{L}_{1}$ norm. Secondly, resorting to introduce auxiliary variables, the primal non-smooth energy functional regularization model without condition constraints is converted into energy functional regularization model with condition constraints, which is split into six easily computing subproblems by the alternating direction method of multipliers (ADMM). Finally, alternating iterative six sub-problems, an improved image reconstruction algorithm is proposed. Numerical experiments show that the proposed model has advantages over several state-of-art approaches in terms of the reconstruction visual effect.
\end{abstract}

\section{Introduction}

In recent years, reconstruction blurred image by system and noise, the widespread acceptance is how to establish hybrid energy functional regularization model, which is composed of fitting term and regularization term [1]. For blurry image by salt and pepper noise, the image statistical distribution is described by $\mathrm{L}_{1}$ norm, the solution singularity property is described by bounded variation (BV) function [2]. However, BV easily leads to texture information of image lost, and produces artificial edges in steady area. For overcoming the disadvantages, reference [3] proposed adaptive energy functional regularization model, but the parameters of weight coefficient are difficult to determine. For reducing the stair effect of steady area of BV, reference [4] used the second BV for regularization term of image reconstruction model. However, the four order elliptic equation is obtained by energy functional regularization model of calculus of variation, which easily smears image edge.

First order BV is advantage of image edges [5], second order BV is advantage of image steady area [6], which shows the complex image characteristics, it is difficult to describe solution properties by a special functional space, hence, hybrid functional space is adopted for description of image properties [7]. Recent research show the image is composed of cartoon and texture information [2], which can be described by different function spaces, and establishment hybrid energy functional regularization model based on image decomposition, which is widely applied in image reconstruction

\footnotetext{
${ }^{a}$ Corresponding author: bsx7096@sohu.com
} 
and compress sensing [8]. For overcoming the image over smooth caused by higher order model, image cartoon part is described by fractional BV [9]. For embodying the sparse property of solution, using tight frame transform [10] for image texture part sparse property, the transform coefficients is described by $\mathrm{L}_{1}$ norm [11].

The rest of this paper is organized as follows : In section 2, we review the tight frame theory and establish hybrid energy functional regularization model for reconstruction image blurred by system and salt and pepper noise. In section 3, taking advantage of the alternative direction method of multiplier (ADMM), we split the hybrid energy functional regularization into six sub-problems, which form alternative iterative algorithm. In section 4 , we carry out several numerical experiments to show that the reconstruction model and algorithm are effective. In section 5, some remarks and future works are discussed.

\section{Hybrid regularization model for image restoration}

\subsection{Tight frame transform for image sparse representation}

Using a cluster of framelet functions for basic function of tight frame, image can be represented by multi-resolution analysis, and the transform coefficients in tight frame domain are sparse, which can embody image singular characteristics, the details about tight frame theory are presented in reference[12, 13]. Suppose that the countable set $\boldsymbol{W} \subset \boldsymbol{L}_{2}(\boldsymbol{R}), \forall \boldsymbol{u} \subset \boldsymbol{L}_{2}(\boldsymbol{R})$, the tight frame must satisfy

$$
\boldsymbol{u}=\sum_{\psi \in W}\langle\boldsymbol{u}, \psi\rangle \psi
$$

Where $\langle\bullet, \bullet\rangle$ represents the inner product of $\boldsymbol{L}_{2}(\boldsymbol{R}), \boldsymbol{W}$ represents the tight frame. The element of framelet system $\psi=\left\{\psi_{1}, \psi_{\searrow} \cdots, \psi_{M}\right\}$ is dilated and shifted, and get tight frame $\boldsymbol{W}(\boldsymbol{\psi}) \subset \boldsymbol{L}_{2}(\boldsymbol{R})$ $\boldsymbol{W}(\boldsymbol{\psi})=\left\{2^{l / 2} \psi_{m}\left(2^{l} \bullet-n\right)\right\}, \psi_{m} \in \psi, \psi_{m}$ is called framelet, $m=1, \cdots, M$. The refinable function $\phi$ and framelet function of tight frame satisfy the following expression

$$
\hat{\phi}(2 \omega)=\hat{h}_{0}(\omega) \hat{\phi}(\omega), \hat{\psi}_{m}(2 \omega)=\hat{h}_{m}(\omega) \hat{\phi}(\omega)
$$

Where $\hat{\phi}, \hat{h}_{0}, \hat{h}_{m}$ is the Fourier transform of $\phi, h_{0}, h_{m}$. According to unitary extension principle [14], the filter coefficients of tight frame $\boldsymbol{W}(\boldsymbol{\psi})$ satisfy the following expression

$$
\hat{h}_{0}(\omega) \overline{\hat{h}_{0}(\omega+\gamma \pi)}+\sum_{m=1}^{M} \hat{h}_{m}(\omega) \overline{\hat{h}_{m}(\omega+\gamma \pi)}=\chi_{\gamma, 0}
$$

Where $\gamma=0,1, \chi_{r, 0}$ is sample function.

\subsection{Using Bayesian principle for hybrid image reconstruction model}

Suppose expectation image $\boldsymbol{f}$ and observation image $\boldsymbol{g}$ obey independent statistical distribution, the imaging system is described by matrix $\boldsymbol{A}$ that is produced by point spread function (PSF). The sample image is blurred by salt and pepper noise, then the conditional probability of sample image is defined as

$$
p(\boldsymbol{g} \mid \boldsymbol{f})=\|\boldsymbol{A} \boldsymbol{f}-\boldsymbol{g}\|_{\mathrm{L}_{1}}
$$


Where, Given ideal image, $p(\boldsymbol{g} \mid \boldsymbol{f})$ represents the conditional probability of observation image. In practical application, it is impossible for user to get the ideal image. To obtain physical variables with practical meaning, supposed that the priori probability of ideal image is $p(\boldsymbol{f})$, using Bayesian principle[15], the maximum posterior probability of ideal image is written as

$$
p(\boldsymbol{f} \mid \boldsymbol{g}) \propto p(\boldsymbol{g} \mid \boldsymbol{f}) \cdot p(\boldsymbol{f})
$$

If the transform coefficients of ideal image is sparse, and ideal image belongs to some kinds of function space, such as variable exponent function space, fractional $\mathrm{BV}$ function space and $\mathrm{L}_{1}$ function space, etc. Supposed the image properties of different function space is independent, we can describe these properties by the following probability distribution function

$$
p(\boldsymbol{f})=\prod_{\tau} p\left(T_{\tau} \boldsymbol{f}\right)
$$

Where $T_{\tau}$ represents function space, and $\tau$ is integer, which represents the number of function space. Substituting (4) and (6) into (5), using $\ln$ likelihood function for both sides of equation, the hybrid energy functional regularization model is written as

$$
\hat{\boldsymbol{f}}=\underset{f}{\operatorname{argmin}}\{\boldsymbol{E}(\boldsymbol{f})\}
$$

Where $E(\boldsymbol{f})=\|\boldsymbol{A} \boldsymbol{f}-\boldsymbol{g}\|_{L_{1}}+\sum_{\tau} \ln p\left(T_{\tau} \boldsymbol{f}\right)$.

In order to using the explicit physical meaning of priori probability, at the same time, the problem of (7) is not too complex, using the cartoon and texture for describing the image properties, which are characterized by fractional order $\mathrm{BV}$ function space and $\mathrm{L}_{1}$ norm of tight frame domain, and the priori probabilities of expectation solution are respectively written as

$$
\begin{aligned}
& -\ln p\left(T_{1} \boldsymbol{f}\right)=|\boldsymbol{W} \boldsymbol{x}|_{L_{1}} \\
& -\ln p\left(T_{2} \boldsymbol{f}\right)=\left|\nabla^{\alpha} \boldsymbol{u}\right|_{1}
\end{aligned}
$$

Where $\boldsymbol{x}$ and $\boldsymbol{u}$ respectively represent texture and cartoon. Substituting (8) and (9) into (7), the hybrid energy functional regularization model for image reconstruction is written as

$$
\underset{f, x, u}{\operatorname{argmin}}\{E(f, \boldsymbol{x}, \boldsymbol{u})\}
$$

Where $E(\boldsymbol{f}, \boldsymbol{x}, \boldsymbol{u})=\|\boldsymbol{A} \boldsymbol{f}-\boldsymbol{g}\|_{\mathrm{L}_{1}}+\alpha|\boldsymbol{W} \boldsymbol{x}|_{L_{1}}+\beta\left|\nabla^{\alpha} \boldsymbol{u}\right|_{\mathrm{L}_{1}}, \alpha$ and $\beta$ are non-negative constant. If $\alpha=0$, formula (10) is image deblurry model of tight frame domain.

\section{Splitting principle and algorithm of image reconstruction model}

\subsection{ADMM algorithm}

Formula (10) is composed of fitting term and regularization term, it is non-smooth and -differential, the fixed point iterative algorithm based on first order gradient and Newton iterative algorithm[16] based on second order gradient can not be applied to (10). According to non-smooth convex optimization theory, the most effective method to compute non-smooth convex function that is composed of two terms is variable splitting technology. The variable splitting technology divide the object function that is composed of two terms into two easy to deal with sub-problems, alternating 
iterative sub-problems form ADMM algorithm[17]. Supposed the optimization object function can be formulated as

$$
\underset{\boldsymbol{h}}{\operatorname{argmin}}\left\{\boldsymbol{E}_{1}(\boldsymbol{h})+\boldsymbol{E}_{2}(\boldsymbol{G h})\right\}
$$

Where, non-smooth convex function $\boldsymbol{E}_{1}: \boldsymbol{R}^{r} \rightarrow \boldsymbol{R}$ and $\boldsymbol{E}_{2}: \boldsymbol{R}^{s} \rightarrow \boldsymbol{R}, \boldsymbol{G} \in \boldsymbol{R}^{s \times r}$. Taking advantage of variable splitting technology, formula (11) splits into two sub-problems that are expressed as follows

$$
\begin{aligned}
& \boldsymbol{h}^{k+1}=\underset{h}{\operatorname{argmin}}\left\{E_{1}(\boldsymbol{h})+\frac{\mu}{2}\left\|\boldsymbol{\gamma}^{k}+\boldsymbol{G} \boldsymbol{h}-\boldsymbol{z}^{k}\right\|_{2}^{2}\right\} \\
& \boldsymbol{z}^{k+1}=\underset{z}{\operatorname{argmin}}\left\{E_{2}(\boldsymbol{z})+\frac{\mu}{2}\left\|\boldsymbol{\gamma}^{k}+\boldsymbol{G} \boldsymbol{h}^{k+1}-\boldsymbol{z}\right\|_{2}^{2}\right\} \\
& \boldsymbol{\gamma}^{k+1}=\boldsymbol{\gamma}^{k}+\left(\boldsymbol{G} \boldsymbol{h}^{k+1}-\boldsymbol{z}^{k+1}\right)
\end{aligned}
$$

Supposed $\hat{\boldsymbol{h}}$ is optimal solution of (11), if the cluster point $\left\{\boldsymbol{h}^{k}\right\}$ is produced by alternating iterative (12)-(14), then $\lim _{k \rightarrow \infty}\left\|\boldsymbol{h}^{k}-\hat{\boldsymbol{h}}\right\|_{2}^{2}=0$.

However, image reconstruction model of (10) is composed of three terms, it is impossible to apply ADMM algorithm directly. Hence, the formula (10) must be improved.

\subsection{Transformation of image reconstruction model}

In order to apply ADMM algorithm to (10), we must transform (10) into similar to standard form formula of (11). Introducing auxiliary variables of $\boldsymbol{A} \boldsymbol{f}=\boldsymbol{\theta}, \boldsymbol{A f}-\boldsymbol{g}=\boldsymbol{z}, \boldsymbol{f}=\boldsymbol{u}+\boldsymbol{x}$, the formula (10) is written as

$$
E(\boldsymbol{f}, \boldsymbol{x}, \boldsymbol{u})=\|z\|_{L_{1}}+\alpha|\boldsymbol{W} \boldsymbol{x}|_{L_{1}}+\beta\left|\nabla^{\alpha} \boldsymbol{u}\right|_{L_{1}}
$$

Set $h_{1}(x)=\alpha\|\boldsymbol{W} \boldsymbol{x}\|, h_{2}(\boldsymbol{z})=\|\boldsymbol{z}\|, h_{3}(\boldsymbol{\theta})=\sigma_{R^{*}}(\boldsymbol{\theta}), g_{1}(\boldsymbol{y})=\beta\left\|\nabla^{\alpha} \boldsymbol{u}\right\|, g_{2}(\boldsymbol{D} \boldsymbol{y})=\sum_{j=1}^{3} h_{j}\left(\boldsymbol{D}_{j} \boldsymbol{y}\right)$, formula (15) is converted into standard form, which is written as

$$
\underset{y}{\operatorname{argmin}}\left\{\mathrm{g}_{1}(\boldsymbol{y})+\mathrm{g}_{2}(\boldsymbol{D} \boldsymbol{y})\right\}
$$

Where $\boldsymbol{y}=\left[\begin{array}{lll}\boldsymbol{u} & \boldsymbol{g} & \boldsymbol{f}\end{array}\right]^{\mathrm{T}}, \boldsymbol{D}=\left[\begin{array}{ccc}-\boldsymbol{I} & \boldsymbol{0} & \boldsymbol{I} \\ \boldsymbol{0} & -\boldsymbol{I} & \boldsymbol{A} \\ \boldsymbol{0} & \boldsymbol{0} & \boldsymbol{A}\end{array}\right]$, set $\boldsymbol{b}=\left[\begin{array}{lll}\boldsymbol{x} & \boldsymbol{z} & \boldsymbol{\theta}\end{array}\right]^{\mathrm{T}}$, then $\boldsymbol{b}=\boldsymbol{D} \boldsymbol{y}$. Applying (12)-(14), formula (16) split into two bigger sub-problems, which are written as

$$
\begin{aligned}
& \boldsymbol{y}^{k+1}=\underset{\boldsymbol{y}}{\operatorname{argmin}}\left\{g_{1}(\boldsymbol{y})+\frac{\boldsymbol{\mu}}{2}\left\|\boldsymbol{d}^{k}+\boldsymbol{D} \boldsymbol{y}-\boldsymbol{b}^{k}\right\|_{2}^{2}\right\} \\
& \boldsymbol{b}^{k+1}=\underset{\boldsymbol{b}}{\operatorname{argmin}}\left\{g_{2}(\boldsymbol{z})+\frac{\mu}{2}\left\|\boldsymbol{d}^{k}+\boldsymbol{D} \boldsymbol{y}^{k+1}-\boldsymbol{b}\right\|_{2}^{2}\right\}
\end{aligned}
$$




$$
\boldsymbol{d}^{k+1}=\boldsymbol{d}^{k}+\left(\boldsymbol{D}^{k+1}-\boldsymbol{b}^{k+1}\right)
$$

1) according to sub-problem of (17), optimization about $\boldsymbol{y}$ is expressed as

$$
\left(\boldsymbol{u}^{k+1}, \boldsymbol{g}^{k+1}, \boldsymbol{f}^{k+1}\right)=\underset{\boldsymbol{u}, \boldsymbol{g}, f}{\operatorname{argmin}}\left\{\beta\left|\nabla^{\alpha} \boldsymbol{u}\right|_{\mathrm{TV}}+\frac{\mu}{2}\left(\left\|\boldsymbol{d}_{1}^{k}+\boldsymbol{f}-\boldsymbol{u}-\boldsymbol{x}^{k}\right\|_{2}^{2}+\left\|\boldsymbol{d}_{2}^{k}+\boldsymbol{A} \boldsymbol{f}-\boldsymbol{g}-\boldsymbol{z}^{k}\right\|_{2}^{2}+\left\|\boldsymbol{d}_{3}^{k}+\boldsymbol{A} \boldsymbol{f}-\boldsymbol{\theta}^{k}\right\|_{2}^{2}\right)\right\}
$$

Optimization (20) about $\boldsymbol{f}, \boldsymbol{u}$ and $\boldsymbol{g}$, respectively, they form the following three sub-problems (1) optimization (20) about $\boldsymbol{f}$ sub-problem

$$
\boldsymbol{f}^{k+1}=\underset{f}{\operatorname{argmin}} \frac{\mu}{2}\left\{\left\|\boldsymbol{d}_{1}^{k}+\boldsymbol{f}-\boldsymbol{u}-\boldsymbol{x}^{k}\right\|_{2}^{2}+\left\|\boldsymbol{d}_{2}^{k}+\boldsymbol{A} \boldsymbol{f}-\boldsymbol{g}-\boldsymbol{z}^{k}\right\|_{2}^{2}+\left\|\boldsymbol{d}_{3}^{k}+\boldsymbol{A} \boldsymbol{f}-\boldsymbol{\theta}^{k}\right\|_{2}^{2}\right\}(21)
$$

Formula (21) is quadratic equation, according to first order Karush-Kuhn-Tucker (KKT) conditions [16], the first order variation of (21) about $\boldsymbol{f}$ is written as

$$
\left(2 \boldsymbol{A}^{\mathrm{T}} \boldsymbol{A}+\boldsymbol{I}\right) \boldsymbol{f}=\boldsymbol{u}+\boldsymbol{x}^{k}-\boldsymbol{d}_{1}^{k}+\boldsymbol{A}^{\mathrm{T}}\left(\boldsymbol{g}+\boldsymbol{z}^{k}-\boldsymbol{d}_{2}^{k}+\boldsymbol{\theta}^{k}-\boldsymbol{d}_{3}^{k}\right)
$$

Imposing periodic boundary condition on image, carrying out Fourier transform and inverse Fourier transform, we can get the following formula

$$
\boldsymbol{f}^{k+1}=F^{-1}\left(\frac{F\left(\boldsymbol{u}^{k}+\boldsymbol{x}^{k}-\boldsymbol{d}_{1}^{k}+\boldsymbol{A}^{\mathrm{T}}\left(\boldsymbol{g}^{k}+\boldsymbol{z}^{k}-\boldsymbol{d}_{2}^{k}+\boldsymbol{\theta}^{k}-\boldsymbol{d}_{3}^{k}\right)\right)}{F\left(2 \boldsymbol{A}^{\mathrm{T}} \boldsymbol{A}+\boldsymbol{I}\right)}\right)
$$

Where $F$ and $F^{-1}$ respectively represent Fourier transform and inverse Fourier transform.

(2) optimization (20) about $\boldsymbol{u}$ sub-problem

$$
\boldsymbol{u}^{k+1}=\underset{u}{\operatorname{argmin}}\left\{\beta \mid \nabla^{\alpha} \boldsymbol{u}_{\mathrm{TV}}+\frac{\boldsymbol{\mu}}{2}\left\|\boldsymbol{d}_{1}^{k}+\boldsymbol{f}-\boldsymbol{u}-\boldsymbol{x}^{k}\right\|_{2}^{2}\right\}
$$

In order to obtain the solution of (24), taking advantage of Fenchel transform for converting (24) into dual model [18], and using fixed point iterative theory, we get the following expression

$$
\boldsymbol{u}^{k+1}=\left(\boldsymbol{d}_{1}^{k}+\boldsymbol{f}^{k+1}-\boldsymbol{u}^{k}-\boldsymbol{x}^{k}\right)-\frac{\beta}{\mu} \overline{(-1)^{\alpha}} \operatorname{div}^{\alpha} \boldsymbol{p}^{k+1}
$$

where $\boldsymbol{p}^{k+1}$ is expressed as

$$
\boldsymbol{p}^{k+1}=\frac{\boldsymbol{p}^{k}+\tau\left[\nabla^{\alpha} \overline{(-1)^{\alpha}} \operatorname{div}^{\alpha} \boldsymbol{p}^{k}-\frac{\beta}{\mu}\left(\boldsymbol{d}_{1}^{k}+\boldsymbol{f}^{k+1}-\boldsymbol{u}^{k}-\boldsymbol{x}^{k}\right)\right]}{1+\tau\left|\nabla^{\alpha} \overline{(-1)^{\alpha}} \operatorname{div}^{\alpha} \boldsymbol{p}^{k}-\frac{\beta}{\mu}\left(\boldsymbol{d}_{1}^{k}+\boldsymbol{f}^{k+1}-\boldsymbol{u}^{k}-\boldsymbol{x}^{k}\right)\right|}
$$

(3) optimization (20) about $\boldsymbol{g}$ sub-problem

$$
\boldsymbol{g}^{k+1}=\underset{g}{\operatorname{argmin}} \frac{\mu}{2}\left(\left\|\boldsymbol{d}_{2}^{k}+\boldsymbol{A} \boldsymbol{f}-\boldsymbol{g}-\boldsymbol{z}^{k}\right\|_{2}^{2}\right)
$$

According to first order KKT conditions, the first order variation of (21) about $\boldsymbol{g}$ is written as 


$$
\boldsymbol{g}^{k+1}=\boldsymbol{A} \boldsymbol{f}^{k+1}-\boldsymbol{z}^{k}+\boldsymbol{d}_{2}^{k}
$$

2)according to sub-problem of (18), optimization about $\boldsymbol{b}$ is expressed as

$$
\begin{gathered}
\left(\boldsymbol{z}^{k+1}, \boldsymbol{x}^{k+1}, \boldsymbol{\theta}^{k+1}\right)=\underset{z, \boldsymbol{\theta}, \boldsymbol{x}}{\operatorname{argmin}}\left\{\|\boldsymbol{z}\|_{1}+\alpha\|\boldsymbol{W} \boldsymbol{x}\|_{1}+\sigma_{R^{*}}(\boldsymbol{\theta})+\frac{\mu}{2}\left(\left\|\boldsymbol{d}_{1}^{k}+\boldsymbol{f}^{k+1}-\boldsymbol{u}^{k+1}-\boldsymbol{x}\right\|_{2}^{2}+\right.\right. \\
\left.\left.\left\|\boldsymbol{d}_{2}^{k}+\boldsymbol{A} \boldsymbol{f}^{k+1}-\boldsymbol{g}^{k+1}-\boldsymbol{z}\right\|_{2}^{2}+\left\|\boldsymbol{d}_{3}^{k}+\boldsymbol{A} \boldsymbol{f}^{k+1}-\boldsymbol{\theta}\right\|_{2}^{2}\right)\right\}
\end{gathered}
$$

Optimization (29) about $\boldsymbol{z}, \boldsymbol{x}$ and $\boldsymbol{\theta}$, respectively, they form the following three sub-problems (4) optimization (20) about $z$ sub-problem

$$
\boldsymbol{z}^{k+1}=\underset{\boldsymbol{z}}{\operatorname{argmin}}\left\{\|\boldsymbol{z}\|_{1}+\frac{\mu}{2}\left\|\boldsymbol{d}_{2}^{k}+\boldsymbol{A} \boldsymbol{f}^{k+1}-\boldsymbol{g}^{k+1}-\boldsymbol{z}\right\|_{2}^{2}\right\}
$$

Formula (30) is standard L2-L1 type, we can get

$$
\boldsymbol{z}^{k+1}=\operatorname{Soft}_{1 / \mu}\left\{\boldsymbol{g}^{k+1}-\left(\boldsymbol{d}_{2}^{k}+\boldsymbol{A} \boldsymbol{f}^{k+1}\right)\right\}
$$

Where $\operatorname{Soft}_{\xi}[\pi]=\max \{0,|\pi|-\xi\} \operatorname{sgn}(\pi), \operatorname{sgn}(\bullet)$ is sign function.

(5) optimization (29) about $\boldsymbol{x}$ sub-problem

$$
\boldsymbol{x}^{k+1}=\underset{\boldsymbol{x}}{\operatorname{argmin}}\left\{\alpha\|\boldsymbol{W} \boldsymbol{x}\|_{1}+\frac{\boldsymbol{\mu}}{2}\left\|\boldsymbol{d}_{1}^{k}+\boldsymbol{f}^{k+1}-\boldsymbol{u}^{k+1}-\boldsymbol{x}\right\|_{2}^{2}\right\}
$$

Similar to (30), we get the following formula

$$
\boldsymbol{x}^{k+1}=\boldsymbol{W}^{\mathrm{T}} \operatorname{Soft}_{\alpha / \mu}\left[\boldsymbol{W}\left(\boldsymbol{d}_{1}^{k}+\boldsymbol{f}^{k+1}-\boldsymbol{u}^{k+1}\right)\right]
$$

Where $\boldsymbol{W}^{\mathrm{T}}$ is inverse transform of $\boldsymbol{W}$, and $\boldsymbol{W}^{\mathrm{T}} \boldsymbol{W}=\boldsymbol{I}$, T represents transpose.

(6) optimization (29) about $\boldsymbol{\theta}$ sub-problem

$$
\begin{aligned}
& \boldsymbol{\theta}^{k+1}=\underset{\boldsymbol{\theta}}{\operatorname{argmin}}\left\{\sigma_{R^{*}}(\boldsymbol{\theta})+\frac{\mu}{2}\left\|\boldsymbol{d}_{3}^{k}+\boldsymbol{A} \boldsymbol{f}^{k+1}-\boldsymbol{\theta}\right\|_{2}^{2}\right\} \\
& \boldsymbol{\theta}^{k+1}=\max \left(0, \boldsymbol{d}_{3}^{k}+\boldsymbol{A} \boldsymbol{f}^{k+1}\right)
\end{aligned}
$$

3)according to formula of (19), auxiliary variable $\boldsymbol{d}$ is updated as following

$$
\begin{aligned}
& \boldsymbol{d}_{1}^{k+1}=\boldsymbol{d}_{1}^{k}+\boldsymbol{f}^{k+1}-\boldsymbol{u}^{k+1}-\boldsymbol{x}^{k+1} \\
& \boldsymbol{d}_{2}^{k+1}=\boldsymbol{d}_{2}^{k}+\boldsymbol{A} \boldsymbol{f}^{k+1}-\boldsymbol{g}^{k+1}-\boldsymbol{z}^{k+1} \\
& \boldsymbol{d}_{3}^{k+1}=\boldsymbol{d}_{3}^{k}+\boldsymbol{A} \boldsymbol{f}^{k+1}-\boldsymbol{\theta}^{k+1}
\end{aligned}
$$

\subsection{ADMM algorithm for image reconstruction}

1) set $k=0, \boldsymbol{u}^{0}, \boldsymbol{g}^{0}, \boldsymbol{f}^{0}$, and the maximum iterative number $N$.

2) compute (23) for $\boldsymbol{f}^{k+1}$, compute (26) for $\boldsymbol{p}^{k+1}$, compute (25) for $\boldsymbol{u}^{k+1}$, compute (28) for $\boldsymbol{g}^{k+1}$. 
3) compute (31)for $\boldsymbol{z}^{k+1}$,compute (35) for $\boldsymbol{x}^{k+1}$, compute (36) for $\boldsymbol{\theta}^{k+1}$, compute auxiliary variables $\boldsymbol{d}_{1}^{k+1}, \boldsymbol{d}_{2}^{k+1}$ and $\boldsymbol{d}_{3}^{k+1}$ by (36), (37) and (38), respectively.

4) if $\left\|E\left(\boldsymbol{f}^{k+1}, \boldsymbol{x}^{k+1}, \boldsymbol{u}^{k+1}\right)-E\left(\boldsymbol{f}^{k}, \boldsymbol{x}^{k}, \boldsymbol{u}^{k}\right)\right\| \leq t$ or $k>N$, jump 5), otherwise, set $k=k+1$, jump 2).

5) output the reconstruction image $\boldsymbol{f}^{k+1}$.

\section{Experiment results and analysis}

Using refinable functon and tight frame with two generators for image sparse, they are shown in Figure 1. Taking advantage of Matlab fspecial function for producing PSF of size $9 \times 9$ and $13 \times 13$, they are shown in figure 2(a) and 2(b), respectively. The figure 3(a) and 4(a) is original Barbara image of size $256 \times 256$ and $512 \times 512$, respectively, and grey level is $0-255$. The relative merits of the algorithm assessed by visual quality and quantitative peak signal-to-noise ratio (PSNR), we compare the restored results of the proposed with those of fast iterative soft threshold algorithm (FISTA) [5], dual model [19] and fast total variation deconvolution (FTVd) [20].

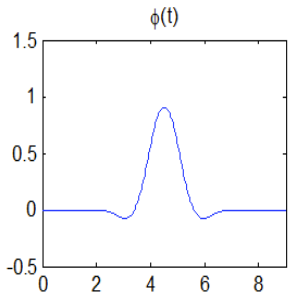

(a) refinable function

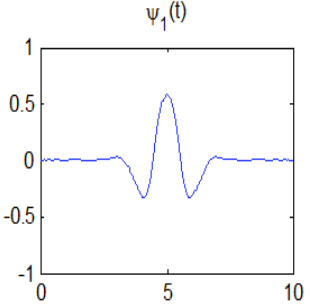

(b) symmetric framelet

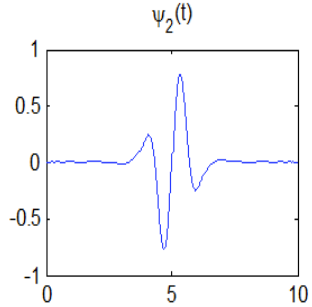

(b) anti-symmetric framelet

Figure 1. refinable function and framelet with two generators
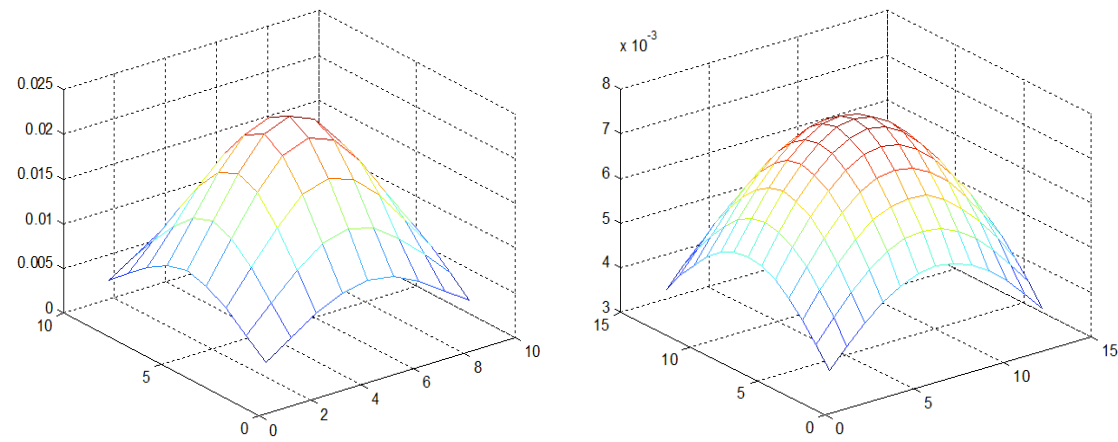

(a) PSF of size $9 \times 9$

(b) PSF of size $13 \times 13$

Figure 2. PSF with different size

In Figure 3, Camera image is blurred by Figure 2(a), and and 30\% salt and pepper noise, it is difficult to distinguish the texture and cartoon information. In Figure 3(c), FISTA makes restored image become cartoon, texture information is completely lost. Dual model and FTVd produce a lot of staircases in steady regions, but restoration image of Figure 3(d) is more smoother than that of Figure 3(e). Figure 3(f), using the proposed for restoration image, the visual effect is better than that of other algorithms. According to qualitative index, PSNR of the proposed is higher that that of other algorithms, which shows image restoration quality is better that of other algorithms. 


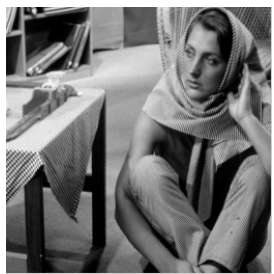

(a) original image

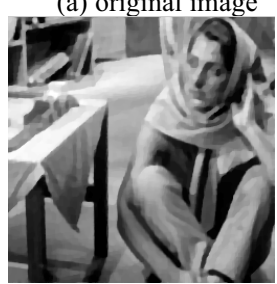

(d) dual model $(26.85 \mathrm{db})$

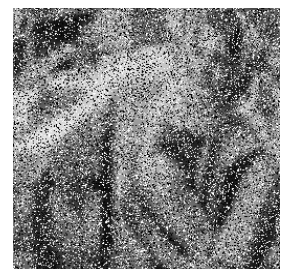

(b) blurry image $(10.03 \mathrm{db})$

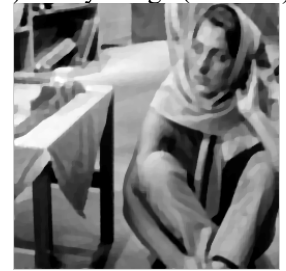

(e) FTVd (27.16db)

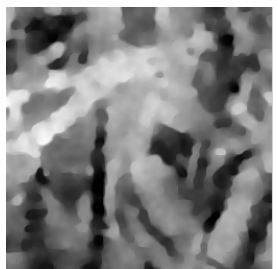

(c) FISTA (17.88db)

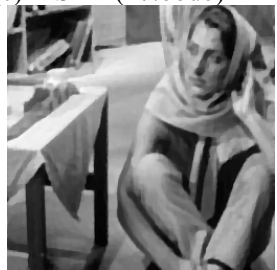

(f) the proposed(27.34db)

Figure 3. Restoration comparison of different algorithms $(256 \times 256)$

Figure 4(b) is is blurred by figure 2(b) of PSF, and $40 \%$ salt and pepper noise, and is more blurry than figure 3(b). According to visual quality, FISTA produces a lot of cartoon, the image texture information is thoroughly lost in figure 4(c). In figure 4(d) and figure 4(e), the visual effect of dual model and FTVd is better than that of FISTA, In figure 4(f), the visual effect is the best in these algorithms.

According to the quantitative performance, FISTA obtains the lowest PSNR, which shows the quality of image restoration is worse. PSNR of dual model is lower than that of FTVd, the proposed obtains the highest PSNR, which show that the quality of image restoration is best.

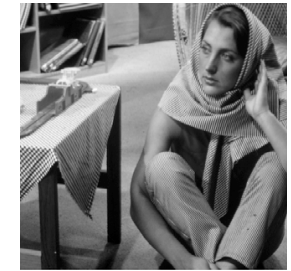

(a) original image

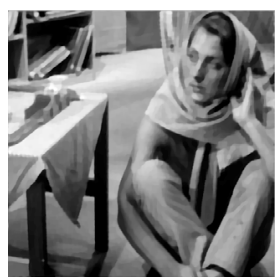

(d) dual model (22.76db)

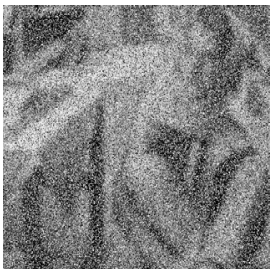

(b) blurry image $(8.78 \mathrm{db})$

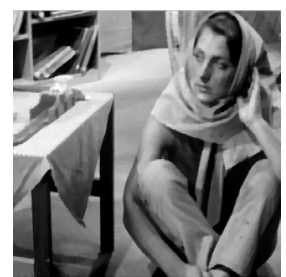

(e) FTVd $(22.99 \mathrm{db})$

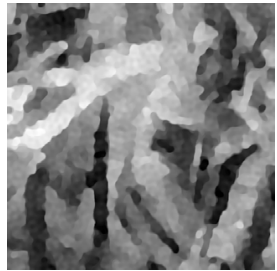

(c) FISTA $(15.89 \mathrm{db})$

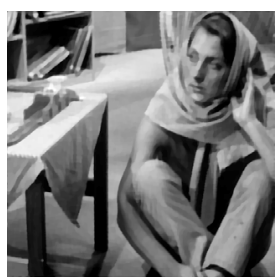

(f) the proposed(23.03db)

Figure 4. Restoration comparison of different algorithms $(512 \times 512)$

\section{Conclusion}

In this paper, a new hybrid energy function regularization model is proposed, by introducing auxiliary variables, image reconstruction model is converted into standard convex optimization model, using ADMM algorithm, standard model is split into six sub-problems, and every sub-problem has simple 
closed expression, which form a fast alternate iterative algorithm. Experiment results show the proposed algorithm can effectively reconstruct image blurred by salt and pepper noise, and obtain better visual quality than that of other methods. In the future work, we will carry out research on the parameters of regularization terms for image restoration performance influence.

\section{Acknowledgement}

This work was supported by Higher Educational Scientific Research Projects of Inner Mongolia Autonomous Region, China (NJZY16254), and project supported by the Natural Science Foundation of Inner Mongolia Autonomous Region, China (2016MS0602).

\section{References}

1. Li X C, Bian S X, Li Y Y, Survey of convex energy functional regularization model of image restoration[J], Journal of Image and Graphics, 21, 4(2016)

2. Zhang Zhengrong, Zhang Jun, Wei Zhihui, Cartoon-texture composite regularization based nonblind deblurring method for partly-textured blurred images with Poisson noise [J], Signal Processing, 116, 11(2015)

3. Li X C, Ma S Y, Bian S X, Application of dual algorithm to TV-L1 deblurring model of frame domain[J], Journal of Image and Graphics, 20, 11(2015)

4. Li Fang, Shen Chaomin, Fan Jingsong, Image restoration combining a total variational filter and a fourth-order filter [J], Journal of Visual Communication and Image Representation, 18, 4(2007)

5. Beck Amir, Teboulle Marc, A fast dual proximal gradient algorithm for convex minimization and applications, Operations Research Letters, 42, 1(2014)

6. Jiang Le, Huang Jin, Lv Xiaoguang, Alternating direction method for the high-order total variation-based Poisson noise removal problem [J], Numerical Algorithms, 69, 3(2015)

7. Fan Qibin, Jiang Dandan, Jiao Yuling. A multi-parameter regularization model for image restoration [J], Signal Processing, 114, 9(2015)

8. Becker Stephen, Bobin Jerome, Candes Emmanuel J, NESTA: a fast and accurate first-order method for sparse recovery [J], SIAM Journal on Imaging Sciences, 4, 1(2011)

9. Yang Z Z, Zhou J L, Lang F N, Noise detection and image de-noising based on fractional calculus [J], Journal of Image and Graphics, 19, 10(2014)

10. Fan W, Cai G G, Zhu Z K, Sparse representation of transients in wavelet basis and its application in gearbox fault feature extraction [J], Mechanical Systems and Signal Processing, 56-57, 5(2015 )

11. Cai Jian-Feng, Ji Hui, Shen Zuowei, Data-driven tight frame construction and image denoising [J], Applied and Computational Harmonic Analysis, 37, 1(2014)

12. Daubechies I, Han B, Ron A, Framelets : mra-based constructions of wavelet frames [J], Applied and Computational Harmonic Analysis, 14, 1(2003)

13. Cai J F, Chan R, Shen Z W, A framelet-based image inpainting algorithm [J], Applied and Computational Harmonic Analysis, 24, 2(2008)

14. Selesnick Ivan W, Abdelnour, Symmetric wavelet tight frames with two generators [J], Applied and Computational Harmonic Analysis, 17, 2(2004)

15. Li X C, Expectation maximization method for parameter estimation of image statistical model[J], Journal of Image and Graphics, 17, 6(2012)

16. Curtis R. Vogel, Computational methods for inverse problems(SIAM: Society for Industrial and Applied Mathematics, 2002)

17. Eckstein J, Bertsekas D P, On the Douglas-Rachford splitting method and the proximal algorithm for maximal monotone operators [J]. Mathematical Programming, 55, 1(1992)

18. Antonin Chambolle, An algorithm for total variation minimization and applications, Journal of Mathematical Imaging and Vision, 20, 1-2(2004) 
19. Clason Christian, Jin Bangti, Kunisch Kral, A duality-based splitting method for 11-tv image restoration with automatic regularization parameter choice, SIAM Journal on Scientific Computing, 32, 3(2010)

20. Yang J, Zhang Y, Yin W, An efficient tv-11 algorithm for deblurring multichannel images corrupted by impulsive noise, SIAM Journal on Scientific Computing, 31, 4(2009) 